\title{
Some characteristics of psychophysical data obtained with a group version of the up-and-down method
}

JOACHIM F, WOHLWILL

CLARK UNIVERSITY

Data are presented to illustrate some peculiarities of a group version of the up-and-down method proposed by Hanley and Zerbolio (1964) for the study of age changes in psychophysical judgments. When applied to the measurement of distance perception, the method fails to reveal the overconstancy bias found in adults by other methods (e.g., adjustment), while producing a highly inflated picture of the inter-S variability. These results, coupled with certain anomalous age trends uncovered in Hanley and Zerbolio's own application of the method to the study of illusions, are attributed to the limitation of each $S$ to a single discrete judgment based on only the briefest exposure to the situation and thus precluding any adaptation to the task.

As is well known, perceptual judgments obtained by the use of traditional psychophysical methods are prone to the influence of a variety of judgmental biases, such as the starting position or anticipation effects, central tendency effects, errors of the standard, etc. Since these blases are apt to interact with the age variable, they pose a particularly serious problem for the psychologist studying the development of perception.

Recognizing this problem, Hanley and Zerbolio(1964, 1965) have espoused a group version of the up-anddown method (also known as the "staircase method"cf., Cornsweet, 1962), which is basically a variant of the method of limits, in which the magnitude of each successive variable presented to $S$ is determined by S's immediately preceding response. In their procedure, each $\mathrm{S}$ makes only a single judgment, for some particular setting of the variable; his response then determines the setting of the variable for the next $S$ (e.g., if for a setting of $V_{x}, S$ gives a judgment of larger than the standard stimulus, the next $S$ is given a setting of $V_{x-i}$, where $i$ is the width of the interval between successive steps in the variable series).

While this version of the up-and-down method, with one judgment per $S$, derives from the original application of this method in the testing of doses of explosives and insecticides, Hanley and Zerbolio (1964, 1965) are misleading in tying its use in psychophysical judgments to such a group procedure. In point of fact, in psychology it has been heretofore utllized almost exclusively on an individual basis, each $\mathrm{S}$ being given an extended series of judgments from which his individual PSE can be determined. Conversely, other psychophysical methods, such as those of limits and constant stimuli, could be adapted to a group procedure analogous to the one proposed by Hanley and Zerbolio, with one judgment per $S$ and a single PSE for the total group resulting. The data thus obtained would presumably be equally free of the biases mentioned by Hanley and Zerbolio, created by presenting $S$ with an extended series of variables or by asking him to adjust the magnitude of the variable himself. The main aim of this note, however, is to point to certain likely consequences of the adoption of such a one-judgment-per-S approach, which is apt to result in data that are not comparable to those obtained by more traditional methods. This point will be illustrated by reference to data on bisection judgments obtained by this wr:ter.

\section{Procedure}

The apparatus and experimental situation was that used in the author's previous research on distance perception (Wohlwill, 1963). Briefly, s looked monocularly into a large rectangular viewing box; S's field of view, brightly lit, consisted essentially of a white cardboard panel showing a randomly textured fileld of black stars (the H-Ran. panel of the abovementioned study was used), on which two red markers were standing, defining a distance of $90 \mathrm{~cm}$ stretching along S's line of sight. A black pointer appeared at some point between the two red markers; its position could be moved by means of an electrically driven mechanism activated by $\mathrm{E}$.

Each S made two types of judgments. The first provided data for the group version of the up-and-down method; accordingly, the pointer was placed in the position determined by the preceding S's responses, and $S$ was asked to look through the eyepiece and to indicate whether the front or the rear marker was closer to the black pointer. If he said "Front," the position of the pointer for the next $S$ was moved back $2.5 \mathrm{~cm}$; if he said "Rear," it was moved forward $2.5 \mathrm{~cm}$. (The setting for the first $\mathrm{S}$ was 1.2 cm in front of the true midpoint.) The $2.5 \mathrm{~cm}$ interval width was selected on the basis of prior information about the standard deviation of S's judgments In this situation (which in previous studies has fluctuated between 2.5 and $4.7 \mathrm{~cm}$ ) bearing in mind Hanley and Zerbolio's recommendation that the ratio of the interval width to $\mathrm{s}$ be between 0.5 and 2.0. 
Following this single judgment, S's PSE was determined directly by the adjustment method, i.e., by having $S$ stop the moving pointer when he thought it was just midway between the two markers. Two such settings were determined, one with the pointer moving away from $S$ (ascending) and one with it moving towards $\mathrm{S}$ (descending).

Ss were 84 college undergraduates.

\section{Results}

The mean constant error for the bisection of the $90 \mathrm{~cm}$ distance, as obtained by the up-and-down method, following the estimation procedure of Dixon and Massey (cf., Hanley \& Zerbolio, 1964) was +0.65 (i.e., the mean perceived midpoint was just slightly to the rear of the objective midpoint). The associated $\mathrm{s}$ was 15.28 , again using Dixon and Massey's formula. The corresponding values for the adjustment method were: $X=+3.8 \mathrm{~cm}, \mathrm{~s}=4.29 \mathrm{~cm}$.

\section{Discussion}

Comparison of the results obtained by the up-anddown method with those obtained by the adjustment method shows, first of all, that the former shows a marked reduction of the bias towards "overconstancy" in the distance bisections of adults which appears under the adjustment method in the present study, as it had in previous work of the author's (e.g., Wohlwill, 1963). More remarkable, perhaps, is the tremendous exaggeration of inter-individual variability reflected in the estimate of the standard deviation (15.28) obtained with the group up-and-down method. Indeed, on the basis of this estimate, the results would have to be discarded, as in flagrant violation of the limits placed by Hanley and Zerbolio on the acceptable values of the $\mathrm{d} / \mathrm{s}$ ratio; as noted above, these limits are between 2.0 and 0.5 . In the present case, using the estimated value of $s$, the ratio would be 0.16 . Yet the $s$ of 4.29 actually determined by the adjustment method, which corresponds closely to the standard deviations found in previous research in this situation, yields a ratio of 0.58 , i.e., within the acceptable limits.

As a check on the validity of Dixon and Massey's formulas utilized in the determination of the $\bar{X}$ and $s$ for the up-and-down data, Ss' PSEs obtained by the adjustment method were arranged in random order, and the Ss were then "rerun" according to the upand-down method in purely hypothetical fashion, utilizing their known PSEs to infer the response they should have given to the setting of the pointer that would have been presented to them. (For instance, if a $S$ with a PSE of 50 was hypothetically presented with a pointer at 45 , his response was scored as "Front" and the next $S$ "presented" with the pointer at 47.5.) This procedure yielded an estimated mean error of $3.2 \mathrm{~cm}$ which is a fairly good approximation to the observed mean of $3.8 \mathrm{~cm}$. More to the point, the estimated value of $s$ on the basis of this hypothetical application of the up-and-down method was 4.16, which is virtually identical with the observed value of 4.29. There is little reason, then, to suspect that the estimation porcedures are at fault.

The explanation for the marked discrepancy between the results for the two methods, both in regard to central tendency and to variability, probably has to do with the peculiar nature of the judgments obtained by the group up-and-down method, in which each $\mathrm{S}$ makes but a single, discrete response, based on a brief inspection of a static stimulus situation. That is to say, there is no opportunity for him to become effectively adapted to the situation, to establish a frame of reference for his judgments, etc. The important role played by these factors, at least in the experimental situation utilized here, is shown in the consistent enhancement of the overconstancy bias from the initial to subsequent bisection judgments found in the author's previous research (Wohlwill, 1963 , 1964), as well as in the marked diminution of the A-D factor (the discrepancy between the values obtained under the ascending and descending conditions of the movement of the pointer), from the first to subsequent judgments. Thus it seems reasonable to suppose that the data obtained from the single, brief exposures to the viewing box with the group up-and -down method reflected to a heightened degree the lack of opportunity for Ss to adapt to the situation, resulting both in highly variable judgments and in the virtual elimination of the average overconstancy bias which normally develops as Ss become adapted and start forming compensatory sets or attitudes.

Clearly these observations do not invalidate the use of Hanley and Zerbolio's procedure, but they do suggest that the results obtained from it must be interpreted with great caution. It is likely, for instance, that in their own application of this method to the investigation of age changes in visual illusions, the failure to replicate the marked reduction of the Muller-Lyer lllusion with age which has almost invariably been found in previous studies (cf., Wohlwill, 1960) has a very similar explanation. There is evidence from research on practice effects and on the effects of limited exposure time that this illusion is at a maximum in the initial phase of $S^{\prime} s$ exposure to the stimulus situation, and that practice effects increase with age (cf., the work of Noelting, discussed by Hanley and Zerbolio). Thus there is a strong possibility that here again the use of the group up-and-down method yields perceptual judgments such as would be obtained in the absence of exposure or adaptation to the situation. The finding of a mean error of almost $35 \%$ in the college group in their study, which is well in excess of the extent of the error found in most previous research with adults, where more tra- 
ditional psychophysical methods have been used, lends further support to this interpretation. In assessing Hanley and Zerbolio's conclusion, therefore, that the use of their method presents a different picture of developmental changes from that shown by the use of the method of adjustment, this characteristic feature of the single-judgment per $S$ procedure must be borne in mind.

Given the unrepresentative nature of some of the results obtained via the use of this method, and the patent impossibility of correlating such judgments with any other information about a given $\mathrm{S}-$ such as judgments on other tasks, measures of intelligence or personality, etc.-its place in the study of psychophysical judgment appears to be limited.

\section{References}

Cornsweet, T. N. The stair-case-method in psychophysics. Amer. J. Psychol., 1962, 75, 485-491.

Hanley, C., \& Zerbolio, D. J. Application of the Up-and-Down method in measuring perceptual judgment. Child Develpm. 1964, 35, 979-984.

Hanley, C.; \& Zerbolio, D. J. Developmental changes in five illusions measured by the Up-and-Down method. Child Develpm. $1965,36,437-452$.

Wohlwill, J. F. Developmental studies of perception. Psychol. Bull., 1960, 57, 249-288.

Wohlwill, J. F. Overconstancy in distance perception as a function of the texture of the stimulus field and other variables. Percept. mot. Skills, 1963, 17, 831-846.

Wohlwill, J. F. Changes in distance judgments as a function of corrected and noncorrected practice. Percept. mot. Skills, 1964, 19, 403-413.

(Accepted for publication September 12, 1967.) 